\title{
An Indoor Mobile Robot Localization Method Based on Information Fusion
}

\author{
Yang Li, Qijin Ji, Yanqin Zhu \\ School of Computer Science and Technology, Soochow University, Suzhou, China
}

Email address:

liyangous@163.com (Yang Li)

\section{To cite this article:}

Yang Li, Qijin Ji, Yanqin Zhu. An Indoor Mobile Robot Localization Method Based on Information Fusion. Internet of Things and Cloud Computing. Vol. 5, No. 3, 2017, pp. 52-58. doi: 10.11648/j.iotcc.20170503.13

Received: June 28, 2017; Accepted: July 10, 2017; Published: August 2, 2017

\begin{abstract}
In order to meet the requirement of positioning accuracy of indoor mobile robot, an indoor localization method based on information fusion is proposed. Firstly, using the Radio frequency identification (RFID) location method to determine the approximate range of the mobile robot's position, in the scope of the current with visual positioning for robot pose information including location coordinates and the deflection Angle; Secondly, using adaptive weighted fusion method to fuse RFID and visual location information; finally, the final result is obtained by Kalman filtering on the location information. The experimental results show that this method can improve the precision of positioning effectively.
\end{abstract}

Keywords: Indoor Localization, RFID, Visual Retrieval, Information Fusion

\section{Introduction}

With the development of robot field, the research of indoor service robot is becoming a hot spot. The robot's selflocalization is the key to realize the function of path planning and autonomous navigation, so it is very important to improve the automation level of robot.

In the past, a variety of approaches for indoor mobile robot localization has been developed. The global positioning system (GPS) is the most widely used positioning system, but because of the disturbance of the GPS signal and the complexity of the indoor environment, it can't meet the requirement of the high precision indoor localization. Therefore, it is urgent to study an indoor positioning method with high positioning precision and stable performance to meet the needs of indoor mobile robot. Researchers at home and abroad have conducted a lot of research on the localization of indoor mobile robots. Radio frequency identification technology (RFID) [1] is a non-contact sensor technology, because of its low price, strong anti-interference and other advantages to become a research hotspot in the field of location. The common RFID indoor positioning system mainly includes LANDMARC, VIRE, SpotON, etc. The LANDMARC system [2] is simple in layout and easy to realize, but the positioning accuracy is not high. In order to improve the positioning accuracy, the system need to set more reference tags, which increase costs and even the phenomenon of RFID signal interference with each other. The VIRE system [3] introduces the concept of virtual reference label, which can get higher positioning precision without adding additional reference tags, but the calculation is more complicated. Reference [4] proposed an RFID and WIFI signal integration of the indoor positioning method, however, because the WIFI signal and RFID signals are affected by multipath effects, the positioning results difficult to achieve satisfactory results. Using vision sensor for indoor location is a new and more advanced localization technology [5-6]. In the reference [7], the authors used Monte Carlo global localization method is combined with image retrieval to transform the localization problem into position probability distribution problem. The positioning accuracy is high, but the image matching time is too long, which affects the real-time performance of the positioning system.

Aiming at the shortage of single sensor positioning method, this paper designs an indoor mobile robot localization method based on RFID and visual information fusion. Firstly, the signal intensity is transformed into space distance by using the RFID signal propagation model, and the initial position of the mobile robot is obtained by maximum likelihood estimation. According to the results of the initial positioning of the robot, the matching range of the image 
database is reduced, and the real-time image is matched with the image database to get location result. Then, the adaptive weighted fusion method is used to correct the RFID and visual location coordinates. Finally, the Kalman filter is processed to further improve the positioning accuracy The experimental results show that the trajectory generated by the method is more consistent with the real path.

Our localization method has been implemented and tested in a real office environment. In this experiment, it has been shown to be able to estimate the position and angle of the robot and can get its trajectory accurately. The method we propose can achieve better performance than a single sensor when encountering serious noise.

The remainder of this paper is organized as follows: Section 2 presents the technique of RFID position by the maximum likelihood method and the technique of visual position by image matching with structural similarity algorithm. In Section 3, we describe how to integrate RFID and visual positioning information. Section 4 presents various experiments and results analysis. Finally, conclusions are provided in Section 5.

\section{Localization Algorithm of Mobile Robot}

\subsection{RFID Positioning Principle}

According to the principle of RFID wireless signal propagation, the magnitude of received signal strength indicator (RSSI) varies with the distance, and it accords with the logarithm-normal distribution propagation model in ideal state. So that the relationship between the distance $d$ between the tag and the reader and the RSSI of the tag received by the reader is as following [8]:

$$
d=10^{\left[P L\left(d_{0}\right)-P L(d)+X_{0}\right] / 10 N}
$$

Where $P L(d)$ is the RSSI received by RFID reader; $P L\left(d_{0}\right)$ is the RSSI received by RFID reader at point $d_{0} ; N$ is path loss exponent related to the environment; $X_{0}$ is Gaussian random variables and set as $0 ; d_{0}$ is the reference distance, usually is $1 \mathrm{~m}$. In known environments, $P L\left(d_{0}\right)$ and $N$ are deterministic, so the value of $\mathrm{d}$ can be obtained by measuring the $P L(d)$.

In order to determine the parameters, four RFID tags were arranged at $1 \mathrm{~m}$ around the RFID reader, measured 50 times for each position, through the curve fitting to get PL (1) = $47.3, \mathrm{~N}=2.37$.

In the actual situation, due to multipath effect, non visual distance transmission, other electronic equipment signals and human interference, it will lead to the RSSI value exist a certain degree of randomness and instability. The Studies show that the RSSI value satisfies the Gaussian distribution at some distance. So the RSSI value is processed by Gaussian filtering. The Gaussian distribution function as following:

$$
f(x)=\frac{1}{\sqrt{2 \pi} \sigma} e^{-\frac{(x-\mu)^{2}}{2 \sigma^{2}}}
$$

Where $\mu=\frac{1}{n} \sum_{i=1}^{n} x_{i} \quad \sigma^{2}=\frac{1}{n-1} \sum_{i=1}^{n}\left(x_{i}-\mu\right)^{2}$

Normally, the choice of the high probability events probability should be greater than 0.6 , according to standard normal distribution probability table when $\mu-\sigma \leq x \leq \mu+\sigma$ the event probability is $68.27 \%$.

After Gaussian filtering, we can get RSSI range is $\mu-\sigma \leq x \leq \mu+\sigma$, this range of RSSI values are all taken out, and then seek the geometric mean, as the final RSSI. And then the maximum likelihood method [9] is used to locate the robot. The maximum likelihood estimation model is shown in Figure 1.

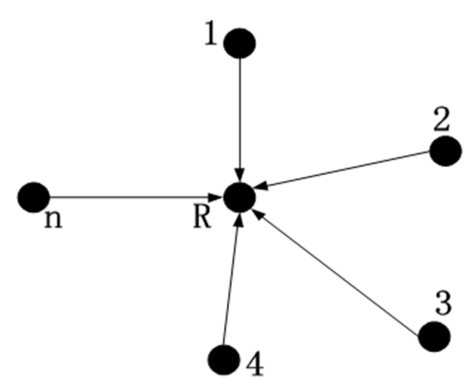

Figure 1. The maximum likelihood estimation model.

The coordinates of the $1,2,3$, and other $\mathrm{n}$ tags are known as $\left(x_{1}, y_{1}\right),\left(x_{2}, y_{2}\right),\left(x_{3}, y_{3}\right),\left(x_{n}, y_{n}\right)$ and the distance between tags and RFID reader $\mathrm{R}$ is $d_{1}, d_{2}, d_{3} \ldots d_{n}$. $\mathrm{R}$ coordinate is $(x, y)$. so:

$$
\left\{\begin{array}{c}
\left(x_{1}-x\right)^{2}+\left(y_{1}-y\right)^{2}=d_{1}^{2} \\
\left(x_{2}-x\right)^{2}+\left(y_{2}-y\right)^{2}=d_{2}^{2} \\
\vdots \\
\left(x_{n}-x\right)^{2}+\left(y_{n}-y\right)^{2}=d_{n}^{2}
\end{array}\right.
$$

The equations in formula (3) are subtracted from the last equation, can get:

$$
\left\{\begin{array}{c}
x_{1}^{2}-x_{n}^{2}-2\left(x_{1}-x_{n}\right) x+y_{1}^{2}-y_{n}^{2}-2\left(y_{1}-y_{n}\right) y=d_{1}^{2}-d_{n}^{2} \\
x_{2}^{2}-x_{n}^{2}-2\left(x_{2}-x_{n}\right) x+y_{2}^{2}-y_{n}^{2}-2\left(y_{2}-y_{n}\right) y=d_{2}^{2}-d_{n}^{2} \\
\vdots \\
x_{n-1}^{2}-x_{n}^{2}-2\left(x_{n-1}-x_{n}\right) x+y_{n-1}^{2}-y_{n}^{2}-2\left(y_{n-1}-y_{n}\right) y=d_{n-1}^{2}-d_{n}^{2}
\end{array}\right.
$$


The formula (4) can be expressed as $A X^{T}=b$, in which:

$$
\begin{gathered}
A=\left[\begin{array}{cc}
2\left(x_{1}-x_{n}\right) & 2\left(y_{1}-y_{n}\right) \\
\vdots & \vdots \\
2\left(x_{n-1}-x_{n}\right) & 2\left(y_{n-1}-y_{n}\right)
\end{array}\right], \\
b=\left[\begin{array}{c}
x_{1}^{2}-x_{n}^{2}+y_{1}^{2}-y_{n}^{2}+d_{n}^{2}-d_{1}^{2} \\
\vdots \\
x_{n-1}^{2}-x_{n}^{2}+y_{n-1}^{2}-y_{n}^{2}+d_{n}^{2}-d_{n-1}^{2}
\end{array}\right], X=\left[\begin{array}{ll}
x & y
\end{array}\right]
\end{gathered}
$$

The position coordinates of $\mathrm{R}$ can be obtained by the least square method:

$$
X=\left(A^{T} A\right)^{-1} A^{T} b
$$

In the positioning process, the reader $\mathrm{R}$ is connected with the mobile robot, and the coordinate of the $\mathrm{R}$ can be obtained by using (5), and it is the coordinate of the robot.

\subsection{The Principle of Visual Positioning}

Visual positioning is divided into off-line phase and online location stage. In the offline stage in the positioning space to build a database connect with pose information (location coordinates and deflection angle) and image information. In the online positioning stage, the real-time image is matched with the image database to obtain the location information..

Image matching algorithm is the key to achieve accurate visual positioning. In this paper, structural similarity (SSIM) algorithm [10] is adopted. The algorithm determines the similarity between images by comparing the contrast, average brightness, and structural information in the two images.

Set $\mathrm{X}$ and $\mathrm{Y}$ be the original image and matching image. Calculate SSIM by the brightness $l(x, y)$, contrast $c(x, y)$ and structural $s(x, y)$. It can be expressed as follows [11]:

$$
\begin{gathered}
l(x, y)=\frac{2 u_{x} u_{y}+C_{1}}{u_{x}^{2}+u_{y}^{2}+C_{1}} \\
c(x, y)=\frac{2 \sigma_{x} \sigma_{y}+C_{2}}{\sigma_{x}^{2}+\sigma_{y}^{2}+C_{2}} \\
s(x, y)=\frac{2 \sigma_{x y}+C_{3}}{\sigma_{x} \sigma_{y}+C_{3}} \\
\operatorname{SSIM}(x, y)=[l(x, y)]^{\alpha} \bullet[c(x, y)]^{\beta} \bullet[s(x, y)]^{\gamma}
\end{gathered}
$$

Where $u_{x}$ and $u_{y}$ is the average brightness value of two pictures. $\sigma_{x}$ and $\sigma_{y}$ is the standard deviation of two picture. $\sigma_{x y}$ is the brightness of the corresponding pixel block correlation coefficient. $C_{1}, C_{2}$ and $C_{3}$ is the tiny constant, to prevent the denominator values close to zero.
SSIM range is $[0,1]$, The larger the value, the higher the similarity of the two images.

\section{Information Fusion Positioning Algorithm}

This paper presents an indoor mobile robot localization method based on RFID and visual information fusion, combining the advantages of RFID and visual positioning technology. First use the characteristics of RFID positioning speed and experience error $\mathrm{E}$ to determine the range of robot. In this range using visual positioning, thereby reduce the matching time. Then use RFID and visual fusion positioning to correct the positioning results so as to minimize the environmental interference. The flow chart of the positioning system is shown in Figure 2.

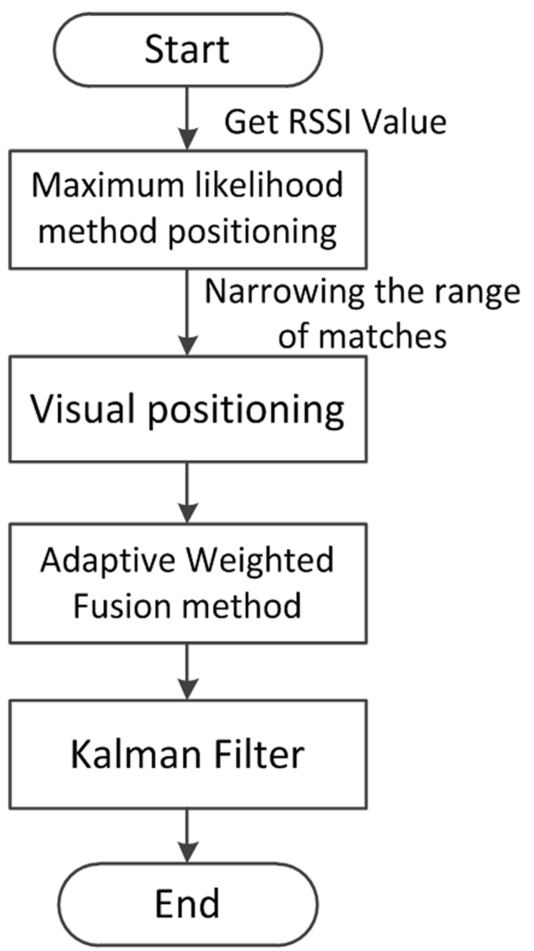

Figure 2. the flow chart of fusion localization algorithm

(1) In this paper, the fusion algorithm is based on the maximum likelihood estimation method based on RFID in the initial stage, The empirical error $\mathrm{E}$ is used to determine the current range of the robot, and the image matching range is reduced for the next step.

(2) Match the real-time image and image database to obtain the matching location result

(3) The adaptive positioning method is used to fuse the RFID positioning result and the visual positioning result, and correct the positioning result.

(4) The Kalman filter [12] is used to further filter the robot position coordinates in order to obtain a smoother trajectory curve. 
The RFID experience error $E$ is calculated after a number of calculations without larger error results, the formula is as following:

$$
E=\frac{1}{N} \sum_{i=0}^{N} \sqrt{\left(x_{i}-X\right)^{2}+\left(y_{i}-Y\right)^{2}}
$$

Where $\mathrm{X}$ and $\mathrm{Y}$ is the real location coordinates. $x_{i}$ and $y_{i}$ is the calculated coordinates, $N$ is the test times.

\subsection{Adaptive Weighted Fusion Algorithm}

In this paper, the adaptive weighted average fusion method combines the RFID and visual positioning results (here does not consider the deflection angle). Through several experiments, we analyzed the data of RFID positioning and visual location in different situations, and assigns different weights to the results of RFID positioning and visual positioning according to different changes of image matching degree, and the larger the image matching degree, the more weight value is given to the visual location result. By fusing two positioning methods, the positioning accuracy can be improved and the interference of environment can be minimized. The Fusion positioning method in this paper is as described in table 1:

\section{Table 1. Adaptive Weighted Fusion Algorithm.}

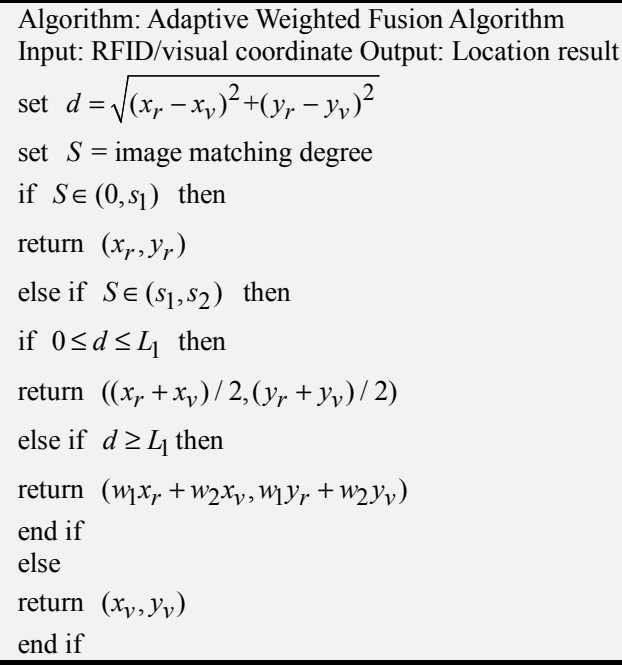

The input of the algorithm is the result of RFID and visual positioning, the output is the final location coordinates, the specific description is as following:

(1) The obtained RFID positioning coordinate is $\left(x_{r}, y_{r}\right)$, the visual position coordinate is $\left(x_{v}, y_{v}\right)$, and the image matching degree is $S$. The distance between the two positioning results is $d=\sqrt{\left(x_{r}-x_{v}\right)^{2}+\left(y_{r}-y_{v}\right)^{2}}$.

(2) If $S$ is located in interval $\left(0, s_{1}\right)$, the location credibility is low because of the low image matching degree. At this time, the RFID positioning result as the positioning output.

(3) If $S$ is located in interval $\left(s_{1}, s_{2}\right)$. At this point, the image matching degree is higher, although the positioning coordinate has the error, but it is within the acceptable range, so it needs to judge the distance $d$. When $0 \leq d \leq L_{1}$, it is determined that the RFID positioning result is not much different from the visual positioning result, and the two position coordinates are averaged to improve the positioning accuracy; When $d \geq L_{1}$, it is determined that the RFID signal is affected by the environment at this time, which leads to the large error of the positioning result. Therefore, the RFID positioning result takes the smaller weight $w_{1}$ and the visual positioning result takes the larger weight $w_{2}$ to reduce the environmental interference. The position of the coordinate point obtained at this time is $(x, y)=\left(w_{1} x_{r}+w_{2} x_{v}, w_{1} y_{r}+w_{2} y_{v}\right)$.

(4) If $S$ is located in interval $\left(s_{2}, 1\right)$, it is can be judged as the image exactly match, the location coordinates is the true coordinates, at this time using visual positioning results as the location output results.

Among them, image matching degree $s_{1}, s_{2}$ and coordinate weights $w_{1}, w_{2}$ is obtained by means of multiple experiments, excluding gross error data after the average calculation.

\subsection{Kalman Fusion Filter Model}

After obtaining the final positioning result, because there is still have noise in the environment, in order to further improve the positioning accuracy, this paper decided to use Kalman filter algorithm to filter the final result. The Kalman filter algorithm consists of several classical core formulas [13] [14]:

(1) Predict the current state:

$$
X(k \mid k-1)=A \bullet X(k-1 \mid k-1)+B \bullet U(k)
$$

Where $X(k \mid k-1)$ is the predict result of previous state. $X(k-1 \mid k-1)$ is the optimal prediction for the last moment. $A$ and $B$ are the system parameters, $U(k)$ is the control of current state, usually be setting as 0 .

(2) Observation equation:

$$
Z(k)=H X(k)+V(k)
$$

Where $Z(k)$ is the observation at $k$ moment. $H$ is the parameter of the measurement system, and the $V(k)$ is the measurement of noise.

(3) Predict error covariance:

$$
P(k \mid k-1)=A \bullet P(k-1 \mid k-1) A^{T}+Q
$$

Where $P(k \mid k-1)$ is the error covariance matrix corresponding to $X(k \mid k-1), P(k-1 \mid k-1)$ is the predictive error covariance matrix corresponding to $X(k-1 \mid k-1), Q$ is the system noise.

(4) Calculate the Kalman gain: 


$$
k g(k)=P(k \mid k-1) \bullet H^{T} /\left(H \bullet P(k \mid k-1) \bullet H^{T}+R\right)
$$

Where $k g(k)$ is the Kalman gain at time $k ; H$ is the gain of the state variable, which is always set as $1 ; R$ is the observed noise.

(5) State estimation

$$
X(k \mid k)=X(k \mid k-1)+k g(k) \bullet(Z(k)-H \bullet X(k \mid k-1))
$$

Where $X(k \mid k)$ is the Kalman filter estimate at time $k$, and $Z(k)$ is the observed value at time $k$.

(6) Update error covariance matrix

$$
P(k \mid k)=(I-k g(k) \bullet H) P(k \mid k-1)
$$

Where $P(k \mid k)$ is the error covariance matrix corresponding to $X(k \mid k), I$ is the unit matrix.

Kalman filter is used in the mobile robot positioning problem. The state to be estimated is the position of the mobile robot, and the abscissa and the ordinate of the robot are moved in the two-dimensional Cartesian coordinate system. The state vector and the observation vector are expressed as:

$$
\begin{gathered}
X(k)=[x(k), y(k)]^{T} \\
Z(k)=\left[Z_{x}(k), Z_{y}(k)\right]^{T}
\end{gathered}
$$

\section{Experimental Results and Analysis}

\subsection{Experimental Conditions}

The experimental site of this paper is an approximately rectangular conference room, about $10 \mathrm{~m}$ long and $8 \mathrm{~m}$ wide. The RFID devices is using the model for Etag-R 701 RFID reader and matching with the Etag-T 701 active tag, this type of RFID reader effective range is about 50 $\mathrm{m} \sim 100 \mathrm{~m}$, it can ensure that all tags in the RFID reader range. Visual positioning using a camera with a pixel of 12 million, in the off-line phase, according to $60 \mathrm{~cm}$ distance interval and 30 degrees of angular interval to collect image data, the entire experimental image database includes about 1200 images.

\subsection{RFID Initial Positioning Error Analysis}

According to the above description of the experimental environment, the tags are arranged in a fixed position, the RFID reader in the indoor space to move the positioning, after several experiments to get the results of positioning error analysis as table 2 .

Table 2. Initial positioning error analysis.

\begin{tabular}{llll}
\hline NO & Real coordinates & experiment coordinates & $\begin{array}{l}\text { Root mean } \\
\text { square error/m }\end{array}$ \\
\hline 1 & $(1,1)$ & $(1.02,0.55)$ & 0.45 \\
2 & $(1.5,1)$ & $(1.22,1.18)$ & 0.33 \\
3 & $(1.5,2)$ & $(0.43,2.1)$ & 1.07 \\
4 & $(2,1)$ & $(1.91,0.85)$ & 0.17 \\
5 & $(3,1.5)$ & $(4.8,1.01)$ & 1.86 \\
6 & $(4,3)$ & $(5.01,3.1)$ & 1.01 \\
7 & $(4.5,3.5)$ & $(4.76,3.86)$ & 0.44 \\
8 & $(4.5,5)$ & $(5.5,4.23)$ & 1.26 \\
9 & $(5,5)$ & $(4.33,5.72)$ & 0.98 \\
10 & $(5,6)$ & $(5.59,8.96)$ & 2 \\
\hline
\end{tabular}

It can be seen from the Table 1, the average error of the indoor positioning based on RFID is $0.96 \mathrm{~m}$. Moreover, the positive and negative errors between true coordinates of the $x-y$ axes and the calculated values are about $0.7 \mathrm{~m}$ after dropping the point whose error is larger. Therefore, in the visual positioning method based on image matching, the matching range of the image database is reduced to $[\mathrm{x} \pm$ $0.7, \mathrm{y} \pm 0.7]$.

\subsection{Comparison of Experimental Results of Information Fusion Location}

In the experimental space, 10 positions are taken for location experiment, and the error analysis of the positioning

\begin{tabular}{|c|c|c|c|c|}
\hline NO & real pose & Measuring position & position error/m & Angle error \\
\hline 1 & $(0.5,4.5,120)$ & $(0.63,4.1,150)$ & 0.42 & 30 \\
\hline 2 & $(1,1,60)$ & $(1.17,1.44,30)$ & 0.22 & 30 \\
\hline 3 & $(1,6,90)$ & $(1.2,6,90)$ & 0.2 & 0 \\
\hline 4 & $(1.5,7,180)$ & $(1.83,7.12,180)$ & 0.35 & 0 \\
\hline 5 & $(2,2.5,60)$ & $(1.74,2.28,30)$ & 0.34 & 30 \\
\hline 6 & $(3.5,4,240)$ & $(3.23,3.65,150)$ & 0.44 & 90 \\
\hline 8 & $(4.5,2,0)$ & $(4.81,2.01,0)$ & 0.31 & 0 \\
\hline 9 & $(5,6,30)$ & $(4.78,5.64,0)$ & 0.42 & 30 \\
\hline 10 & $(5,4,90)$ & $(5.03,3.65,90)$ & 0.35 & 0 \\
\hline
\end{tabular}
results is given as shown in table 3 . The positioning result obtained in this article is set to a three tuple containing coordinate and deflection angles.

Table 3. Error analysis of coordinate and angle location.

It can be seen from the table that the average error of the corrected positioning coordinates after an adaptive weighted fusion is $0.37 \mathrm{~m}$, angle positioning error of 24 degrees
In the experimental space, with a certain trajectory to run, at a certain distance to locate, this fusion method of positioning in the RFID positioning, visual positioning results for 
trajectory comparison, as shown in Figure 3:

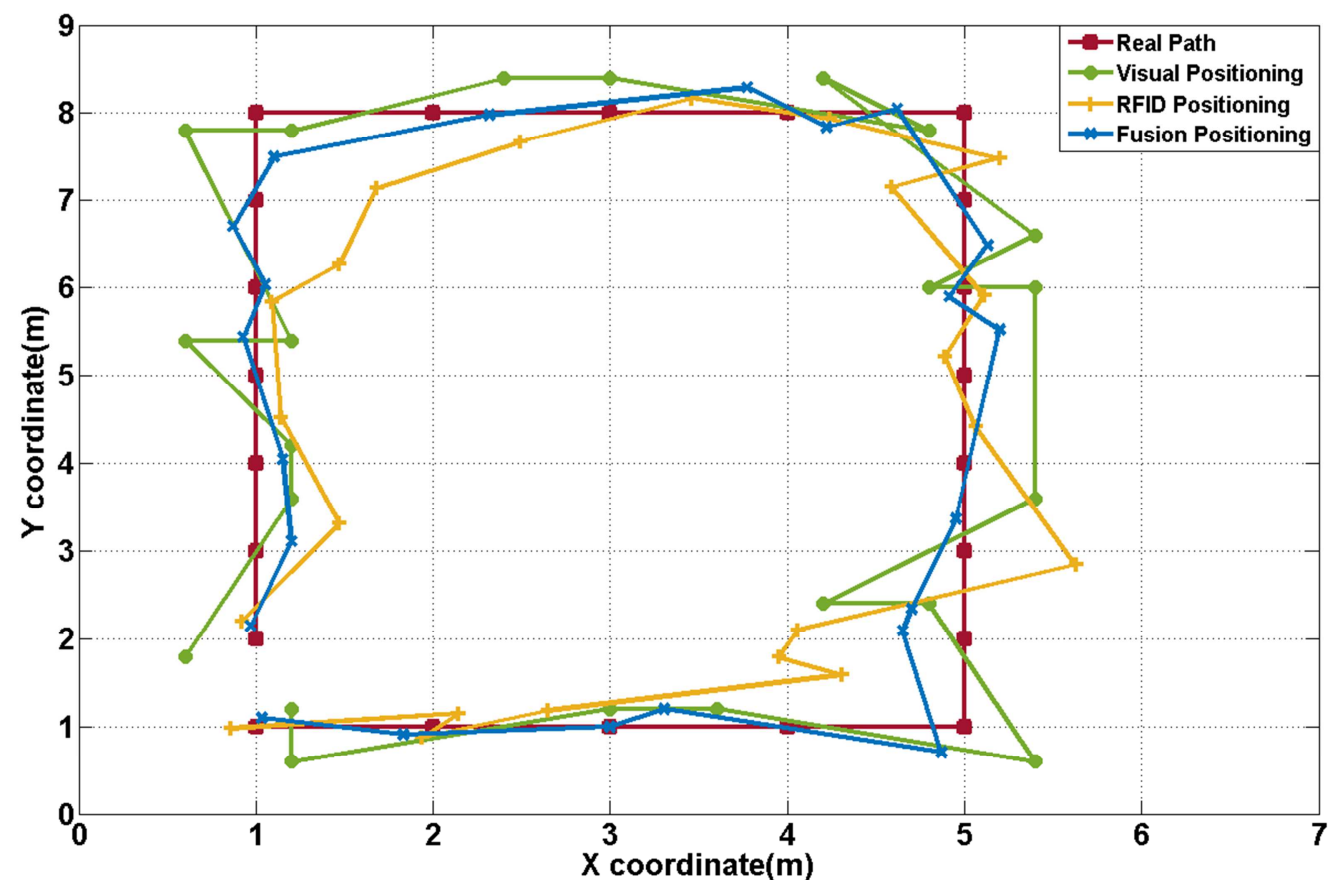

Figure 3. The trajectory comparison of different location methods.

It can be seen from the Fig 3 that the motion trajectory of the fusion location algorithm is obviously closer to the actual motion trajectory, and higher positioning accuracy. It can meet the needs of indoor mobile robot positioning.

\section{Conclusion}

Based on the research and improvement of the existing fusion positioning technology of indoor mobile robots, this paper proposes a positioning method based on information fusion, using adaptive weighted averaging method and Kalman filter to fuse RFID and visual location information. In the experiment, the positioning method of this paper is compared with the single RFID location and the visual location technology. The experimental results show that the trajectory generated by this method is more consistent with the real path, and the positioning accuracy is significantly higher than that of a single positioning method, and the positioning error is $0.37 \mathrm{~m}$, the angle error is 24 degrees in the current experimental environment.

But because of the limited experimental environment, this positioning method is only valid in the smaller space conference room. The next step is to apply this method to the larger space to further verify the universality and positioning accuracy of this method.

\section{References}

[1] Eslim L M, Ibrahim W M, Hassanein H S. GOSSIPY: A distributed localization system for Internet of Things using RFID technology [C]. GLOBECOM 2013 - 2013 IEEE Global Communications Conference. IEEE, 2013, pp. 140-145.
[2] Ni L M, Liu Y, Lau Y C, et al. LANDMARC: indoor location sensing using active RFID [J]. Wireless Networks, 2004, 10 (6), pp. 407.

[3] Zhao Y, Liu Y, Ni L M. VIRE: Active RFID-based Localization Using Virtual Reference Elimination [C]. International Conference on Parallel Processing. IEEE Computer Society, 2007, pp. 56-62.

[4] Potgantwar A D, Wadhai V M. Location Based System for Mobile Devices with Integration of RFID and Wireless Technology-Issues and Proposed System [C]. International Conference on Process Automation, Control and Computing. IEEE, 2011, pp. 1-5.

[5] Bonin-Font F, Ortiz A, Oliver G. Visual Navigation for Mobile Robots: A Survey [J]. Journal of Intelligent \& Robotic Systems, 2008, 53 (3), pp. 263-296.

[6] $\mathrm{Wu} \mathrm{C} \mathrm{J,} \mathrm{Tsai} \mathrm{W} \mathrm{H.} \mathrm{Location} \mathrm{estimation} \mathrm{for} \mathrm{indoor} \mathrm{autonomous}$ vehicle navigation by omni-directional vision using circular landmarks on ceilings [J]. Robotics \& Autonomous Systems, 2009, 57 (5), pp. 546-555.

[7] Wolf J, Burgard W, Burkhardt H. Robust vision-based localization by combining an image-retrieval system with Monte Carlo localization [J]. IEEE Transactions on Robotics, 2005, 21 (2), pp. 208-216.

[8] Wong S F, Ni X. Signal propagation model calibration under metal noise factor for indoor localization by using RFID [C]. IEEE International Conference on Industrial Engineering and Engineering Management. IEEE, 2014, pp. 978-982.

[9] Aso M, Saikawa T, Hattori T. Mobile station location estimation using the maximum likelihood method in sector cell systems [C]. Vehicular Technology Conference, 2002. Proceedings. Vtc 2002-Fall. 2002 IEEE. IEEE Xplore, 2002, pp. 1192-1196. 
[10] Al-Najjar Y. Comparison of Image Quality Assessment: PSNR, HVS, SSIM, UIQI [J]. International Journal of Scientific \& Engineering Research, 2012, 3 (3).

[11] Wang c, Shang Y Y Ding H, et al. Licence plate recognition algorithm research based on SSIM [J]. Optical technique, 2013, 39 (6), pp. 505-509.

[12] Welch G, Bishop G. An Introduction to the Kalman Filter [J]. University of North Carolina at Chapel Hill, 1995 (7), pp. 127-132.
[13] Li Q Y, Chu J K, Li R Q, et al. Moving object tracking algorithm for mobile robot based on Kalman filter [J]. Transducer and Microsystem Technologies, 2008, 27 (11), pp. 66-68.

[14] Chung H Y, Hou C C, Chen Y S. Indoor Intelligent Mobile Robot Localization Using Fuzzy Compensation and Kalman Filter to Fuse the Data of Gyroscope and Magnetometer [J]. IEEE Transactions on Industrial Electronics, 2015, 62 (10), pp. 6436-6447. 